\title{
MEK1 gene mutation in Japanese lung adenocarcinoma patients
}

\author{
HIDEFUMI SASAKI, YU HIKOSAKA, KATSUHIRO OKUDA, OSAMU KAWANO, HARUHIRO YUKIUE, \\ MOTOKI YANO and YOSHITAKA FUJII
}

Department of Surgery II, Nagoya City University Medical School, Nagoya 467-8601, Japan

Received October 23, 2008; Accepted December 9, 2008

DOI: 10.3892/mmr_00000076

\begin{abstract}
Recently, to identify potential somatic mutations in genes of the epidermal growth factor receptor (EGFR) signaling pathway, the MEK1 gene mutation at exon 2 was identified. The mutant form of MEK1 leads to the constitutive activity of extracellular signal-regulated kinase (ERK)-1/2. We investigated MEK1 gene mutation status in 241 surgically treated lung adenocarcinoma cases from Nagoya City University Hospital. The presence or absence of the MEKI mutation was analyzed by direct sequencing. EGFR mutation status was previously investigated and reported. We detected only one case $(0.4 \%)$ of the $M E K 1$ mutation $(\mathrm{K} 57 \mathrm{~N})$ in our cohort. Total EGFR mutations were present in 101 patients (41.9\%). The MEK1 mutation was mutually exclusive with $B$-raf, $K$-ras and EGFR mutations. Thus, it is a rare mutation in Japanese lung cancer patients, and of limited value for lung adenocarcinoma.
\end{abstract}

\section{Introduction}

Lung cancer is a major contributer to malignant disease mortality due to its high incidence, malignant behavior, and the lack of major advancements in treatment strategy (1). The disease was the leading indication for respiratory surgery (42.2\%) in 1998 in Japan (2), with more than 15,000 patients undergoing surgical procedures for lung cancer at Japanese institutions that year (2).

The mitogen-activated protein kinase (MAPK) pathway plays a critical role in the epidermal growth factor receptor (EGFR) signaling cascade. After the activation of EGFR signaling, key downstream steps involve the phosphorylation by RAF1 kinase of two distinct serine residues on both MEK1 and MEK2, upstream of the ERK1/ERK2 MAPK module (3). MEK1 is the main activator of both ERK1 and ERK2, as well as the critical isoform regulating tumor cell proliferation in vitro and in vivo (4). Approximately $25 \%$ of individuals

Correspondence to: Dr Hidefumi Sasaki, Department of Surgery II, 1 Kawasumi, Mizuho-cho, Mizuho-ku, Nagoya 467-8601, Japan E-mail: hisasaki@med.nagoya-cu.ac.jp

Key words: MEK1, lung adenocarcinoma, epidermal growth factor receptor, exon 2, K57N with Cardiofaciocutaneous syndrome (CFC) have mutations in either the $M E K 1$ or $M E K 2$ gene. These lead to increased MEK kinase activity, as judged by increased phosphorylation of its downstream effector ERK $(5,6)$. Germline mutations of MEK are sensitive to MEK and RAF inhibition, suggesting the need for therapeutic options (7). More recently, the MEK1 mutation at exon 2 was identified by mutational analysis of the EGFR signaling pathway genes in lung adenocarcinoma (8). MEK1 mutations at exon 2 were also reported in cell lines, such as rat fibroblasts (9) and the ovarian cancer cell line ES-2 (10).

To determine MEK1 status in Japanese lung adenocarcinoma patients for screening purposes, we investigated $M E K 1$ exon 2 mutation status by direct sequencing. The findings were compared to the clinicopathologic features of the lung adenocarcinoma patients.

\section{Patients and methods}

Patients. The study group included 241 lung adenocarcinoma patients who had undergone surgery at the Department of Surgery II, Nagoya City University Medical School, between 1997 and 2006. We started with 248 samples; however, 7 were defined as non-adenocarcinomas (4 squamous cell carcinomas and 3 adenosquamous carcinomas) upon retrospective pathological review. These 7 samples were $M E K 1$ wild-type (data not shown). Lung tumors were classified according to the general criteria for clinical and pathological recording of lung cancer in Japan. All tumor samples were immediately frozen and stored at $-80^{\circ} \mathrm{C}$ until assayed.

The clinical and pathological characteristics of the 241 lung adenocarcinoma patients were as follows: 151 cases at stage I, 31 at stage II and 59 at stage III-IV. The mean patient age was 64.8 years (range 38-83). Of the 241 lung adenocarcinoma patients, $139(57.7 \%)$ were male and $102(42.3 \%)$ were non-smokers. The samples from these patients had previously been sequenced for EGFR (11-14).

PCR assays for MEK1. Genomic DNA was extracted using the Wizard SV Genomic DNA Purification System (Promega) according to the manufacturer's instructions. We then used 100 ng of each DNA for PCR analyses. The PCR reactions were performed using LA-Taq Kit (Takara Bio Inc., Shiga, Japan) in a $50-\mu 1$ reaction volume. The primer sequences for the $M E K 1$ gene, exon 2 were as follows: forward 5-TTTCTT TCCATGATAGGAGT-3, reverse 5-ATCAGTCTTCCTTCT ACCCT-3. Cycling conditions were as follows: initial denatu- 
ration at $94^{\circ} \mathrm{C}$ for $5 \mathrm{~min}$ followed by 35 cycles at $94^{\circ} \mathrm{C}$ for $45 \mathrm{sec}, 59^{\circ} \mathrm{C}$ for $45 \mathrm{sec}$ and $72^{\circ} \mathrm{C}$ for $30 \mathrm{sec}$. The products were purified using the Qiagen PCR Purification Kit (Qiagen, Valencia, CA). Amplified DNA were separated on $1 \%$ agarose gels, and the bands were visualized by ethidium bromide and photographed under ultraviolet transillumination. These samples were sequenced using the ABI PRISM 3100 analyzer (Applied Biosystems Japan Ltd., Tokyo, Japan) and analyzed by BLAST and chromatograms by manual review.

Statistical analysis. Statistical analyses were carried out using the Mann-Whitney U test for unpaired samples and Wilcoxon's signed rank test for paired samples. Linear relationships between variables were determined by means of simple linear regression. Correlation coefficients were determined by rank correlation using the Spearman and $\chi^{2}$ tests. The overall survival of lung cancer patients was examined by the Kaplan-Meier method, and differences were examined by the Log-rank test. Analyses were performed using the Stat-View software package (Abacus Concepts Inc., Berkeley, CA), and differences were considered significant at $\mathrm{p}$-values $<0.05$.

\section{Results}

MEKI gene mutation status in Japanese lung adenocarcinoma patients. Using the primer sets for MEK1 exon 2, we visualized the PCR products with $1 \%$ agarose gel. These samples were further studied. In our cohort, 1 of 241 patients had the MEK1 mutation K57N (Fig. 1). The patient was male and a smoker. In this analysis, 101 patients had the EGFR mutation at the kinase domain (11-14). The $M E K 1$ mutation was mutually exclusive with $B$-raf (15), K-ras (16) and EGFR mutations.

Retrospective pathological review demonstrated that the MEK1 mutant sample was adenocarcinoma (poorly differentiated) with mixed subtype, $60 \%$ solid and $40 \%$ acinar components. The patient succumbed to the disease 5 months after surgery due to recurrence. Seven of the non-adenocarcinoma samples (4 squamous cell and 3 adenosquamous carcinomas) were $M E K 1$ wild-type (data not shown).

\section{Discussion}

The findings of the present study reveal MEK1 K57N mutation status to be rare in our Japanese cohort $(0.4 \%)$, and mutually exclusive with Braf, $K$-ras and EGFR mutations in adenocarcinoma patients.

Altered MEK proteins have been reported to be implicated in cancer. A transformation-competent mutant form of MEK1 with a Q56P (exon 2) substitution in the non-kinase portion has been described in rat fibroblasts (9), and an engineered mutant with alteration of the key regulatory serine residues is capable of transforming NIH 3T3 cells (17). A human ovarian cancer cell line, ES-2, was found to harbor an activating mutation in $M E K 1$ that substitutes asparagines for aspartic acid at position 67 (G199A, D67N; exon 2) (10). Functional characterization of the MEK1 mutant D67N by transient transfection with subsequent Western blot analysis demonstrated increased ERK phosphorylation as compared to controls (10). Using Tet-inducible shRNA, MEK1 was the main activator

\section{MEK1 sequence}

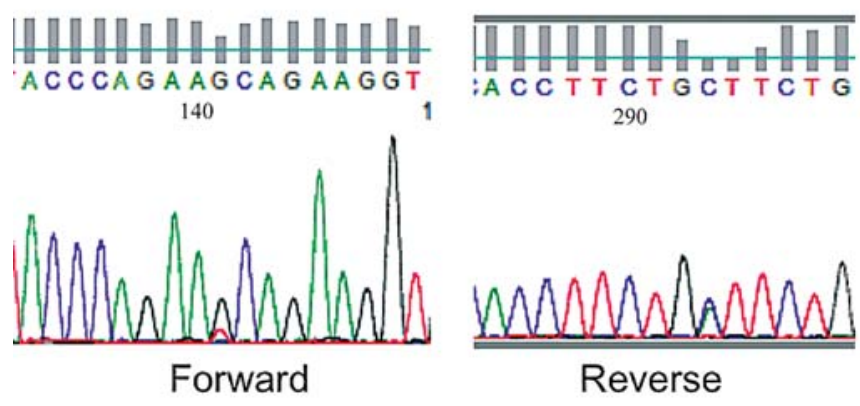

Figure 1. The $M E K 1$ gene mutation. The position at 171 nucleotide change from $\mathrm{G} \rightarrow \mathrm{T}$ at exon 2 introduces a substitution of lysine $(\mathrm{K})$ to asparagine $(\mathrm{N})$ at amino acid position $57(\mathrm{~K} 57 \mathrm{~N})$ of the MEK1 protein.

of both ERK1 and ERK2. In contrast, MEK2 removal had no impact on its own, but did cooperate with MEK1 ablation for the inhibition of ERK1/2 activity in LS174T colon carcinoma cells (4). Small molecule inhibitors of MEK would seem to be promising antitumor agents (18). In addition, a previous study exhibited the somatic mutation in $M E K 1$ in human lung tumors, but not in colon, breast, prostate and pancreatic carcinomas, identified via mutational profiling of the genes encoding EGFR signaling pathway proteins in a large cohort of lung adenocarcinomas $(8,19)$.

In a previous study, the same heterozygous mutation, K57N, was found in 2 of 207 lung adenocarcinomas. Neither of these two harbored mutations in other genes encoding components of the EGFR signaling pathway, such as EGFR, $K$-ras, $B$-raf or PIK3CA (8). Functional characterization of the mutant form in vitro indicated that its expression in $293 \mathrm{~T}$ cells leads to constitutive activation of downstream signaling components (9). Thus, the MEK1 K57N mutant form displays gain-of-function properties (9). K57 is highly conserved among various species (8), and is located in a region between the nuclear export signal (amino acids 33-44) and the catalytic domain (amino acids 68-271) of MEK1. Consistent with the $\mathrm{G} \rightarrow \mathrm{T}$ mutation (a type of transversion known to be smoking-related), the sample was from a smoker. The K57N substitution led to constitutive activation of the MAPK pathway in vitro ( $\mathrm{Ba} / \mathrm{F} 3$ cells) (8).

A previous study identified germ-line $M E K 1$ mutations in patients with CFC syndrome, a complex developmental disorder involving the heart, face and skin (5). Approximately $25 \%$ of individuals with CFC have mutations in either MEK1 or $M E K 2$, which lead to increased MEK kinase activity, as judged by increased phosphorylation of its downstream effector MEK (6). These mutations include F53S, G128V and Y130C MEK1. The latter two of these occur in the kinase domain $(5,20)$. The F53S mutant, like the Y130C mutant, is more active than wild-type protein in stimulating ERK phosphorylation (5). It is not yet clear if CFC patients are at an increased risk of cancer, but three affected individuals have developed neoplasms (21-23).

In summary, the MEK1 mutation may play a role in EGFR signaling. However, it is rare in Japanese lung cancer patients, and of limited value for lung adenocarcinoma. 


\section{Acknowledgements}

We thank Mrs. Mayu Uemura for her excellent technical assistance. The study was sponsored by a Grand-in-Aid for Research by Nagoya City University (2006) and Grants-inAid for Scientific Research, Japan Society for the Promotion of Science (JSPS) (nos. 19390367, 18390381, 18659407).

\section{References}

1. Ginsberg RJ, Kris $\mathrm{K}$ and Armstrong G: Cancer of the lung. In: Principles and Practice of Oncology. 4th edition, Lippincott, Philadelphia, pp673-682, 1993.

2. Yasuda K, Ayabe H, Ide $H$ and Uchida $Y$ : On behalf of the Japanese Association for Thoracic Surgery: Thoracic and cardiovascular surgery in Japan during 1998. Annual report by the Japanese Association for Thoracic Surgery. Jpn J Cardiothorac Surg 48: 401-415, 1998 .

3. Kyriakis JM, App H, Zhang XF, et al: Raf-1 activates MAP kinase-kinase. Nature 358: 417-421, 1992.

4. Shama J, Garcia-Medina R, Pouyssegur J and Vial E: Major contribution of MEK1 to the activation of ERK1/ERK2 and to the growth of LS174T colon carcinoma cells. Biochem Biophys Res Commun 372: 845-849, 2008.

5. Rodriguez-Viciana P, Tetsu O, Tidyman WE, et al: Germline mutations in genes within the MAP pathway cause cardio-faciocutaneous syndrome. Science 3: 1287-1290, 2006.

6. Rodriguez-Viciana P and Rauen KA: Biochemical characterization of novel germline BRAF and MEK mutations in cardiofaciocutaneous syndrome. Methods Enzymol 438: 277-289, 2008.

7. Senawong T, Phuchareon J, Ohara O, McCormick F, Rauen KA and Tetsu O: Germline mutations of MEK in cardio-faciocutaneous syndrome are sensitive to MEK and RAF inhibition: implications for therapeutic options. Hum Mol Genet 17: 419-430, 2008.

8. Marks JI, Gong Y, Chitale D, et al: Novel MEK1 mutation identified by mutational analysis of epidermal growth factor receptor signaling pathway genes in lung adenocarcinoma. Cancer Res 68: 5524-5528, 2008.

9. Bottorff D, Stang S, Agellon S and Stone JC: RAS signaling is abnormal in a c-raf1 MEK1 double mutant. Mol Cell Biol 15: 5113-5122, 1995.
10. Estep AL, Palmer C, McCormick F and Rauen KA: Mutation analysis of BRAF, MEK1 and MEK2 in 15 ovarian cancer cell lines: implications for therapy. PLoS ONE 2: e1279, 2007.

11. Paez JG, Janne PA, Lee JC, et al: EGFR mutations in lung cancer: correlation with clinical response to gefitinib therapy. Science 304: 1497-1500, 2004.

12. Endo K, Konishi A, Sasaki H, et al: Epidermal growth factor receptor gene mutation in non-small cell lung cancer using highly sensitive and fast TaqMan PCR assay. Lung Cancer 50: 375-384, 2005

13. Sasaki H, Shimizu S, Endo K, et al: EGFR and erbB2 mutation status in Japanese lung cancer patients. Int J Cancer 118 : 180-184, 2006.

14. Sasaki H, Endo K, Konishi A, et al: EGFR mutation status in Japanese lung cancer patients: genotyping analysis using LightCycler. Clin Cancer Res 11 : 2924-2929, 2005.

15. Sasaki H, Kawano O, Endo K, et al: Uncommon V599E BRAF mutations in Japanese patients with lung cancer. J Surg Res 133: 203-206, 2006.

16. Sasaki H, Okuda K, Kawano O, et al: Nras and Kras mutation in Japanese lung cancer patients: Genotyping analysis using LightCycler. Oncol Rep 18: 623-628, 2007.

17. Mansour SJ, Matten WT, Hermann AS, et al: Transformation of mammalian cells by constitutively active MAP kinase kinase. Science 265: 966-970, 1994.

18. Sebolt-Leopold JS, Dudley DT, Herrera R, et al: Blockade of the MAP kinase pathway suppress growth of colon tumors in vivo. Nat Med 5: 810-816, 1999.

19. Marks JL, McLellan MD, Zakowski MF, et al: Mutational analysis of EGFR and related signaling pathway genes in lung adenocarcinomas identifies a novel somatic kinase domain mutation in FGFR4. Prot PLosS ONE 2: e426, 2007.

20. Schulz AL, Alberecht B, Arici C, et al: Mutation and phenotypic spectrum in patients with cardio-ficio-cutaneous and Costello syndrome. Clin Genet 73: 62-70, 2008.

21. Bisogno G, Murgis A, Mimmi I, Strafella MS and Carli M: Rhabdomyosarcoma in a patient with cardio-facio-cutaneous syndrome. J Pediatr Hematol Oncol 21: 424-427, 1999.

22. Al-Rahawan MM, Chute DJ, Sol-Church K, et al: Hepatoblastoma and heart transplantation in a patient with cardiofacio-cutaneous syndrome. Am J Med Genet A 143: 1481-1486, 2007.

23. Makita Y, Narumi Y, Yoshida M, et al: Leukemia in Cardiofacio-cutaneous (CFC) syndrome: a patient with a germline mutation in BRAF proto-oncogene. J Pediatr Hematol Oncol 29: 287-290, 2007. 